\title{
Molecular signatures of age-associated chronic degeneration of shoulder muscles
}

\author{
Yotam Raz ${ }^{1,2,3, *}$, Jan Ferdinand Henseler ${ }^{1, *}$, Arjen Kolk ${ }^{1}$, Zuotian Tatum ${ }^{2}$, Niels \\ Kuipers Groosjohan ${ }^{2}$, Nisha E. Verwey², Wibowo Arindrarto ${ }^{3}$, Szymon M. Kielbasa ${ }^{3}$, \\ Jochem Nagels', Peter A. C. 't Hoen'², Rob G. H. H. Nelissen'1 and Vered Raz ${ }^{2}$ \\ ${ }^{1}$ Department of Orthopaedics, Leiden University Medical Center, Leiden, The Netherlands \\ 2 Department of Human Genetics, Leiden University Medical Center, Leiden, The Netherlands \\ ${ }^{3}$ Department of Medical Statistics and Bioinformatics, Leiden University Medical Center, Leiden, The Netherlands \\ * Equal first authorship \\ Correspondence to: Vered Raz, email: v.raz@lumc.nl \\ Keywords: shoulder disease, atrophy, fatty infiltration, muscle satellite cells, deep RNA-seq, Gerotarget \\ Received: January 08, $2016 \quad$ Accepted: January 30, $2016 \quad$ Published: February 14, 2016
}

\section{ABSTRACT}

Chronic muscle diseases are highly prevalent in the elderly causing severe mobility limitations, pain and frailty. The intrinsic molecular mechanisms are poorly understood due to multifactorial causes, slow progression with age and variations between individuals. Understanding the underlying molecular mechanisms could lead to new treatment options which are currently limited. Shoulder complaints are highly common in the elderly, and therefore, muscles of the shoulder's rotator cuff could be considered as a model for chronic age-associated muscle degeneration. Diseased shoulder muscles were characterized by muscle atrophy and fatty infiltration compared with unaffected shoulder muscles. We confirmed fatty infiltration using histochemical analysis. Additionally, fibrosis and loss of contractile myosin expression were found in diseased muscles. Most cellular features, including proliferation rate, apoptosis and cell senescence, remained unchanged and genome-wide molecular signatures were predominantly similar between diseased and intact muscles. However, we found down-regulation of a small subset of muscle function genes, and up-regulation of extracellular region genes. Myogenesis was defected in muscle cell culture from diseased muscles but was restored by elevating MyoD levels. We suggest that impaired muscle functionality in a specific environment of thickened extra-cellular matrix is crucial for the development of chronic age-associated muscle degeneration.

\section{INTRODUCTION}

Aging-associated muscle degeneration leads to functional impairments of daily activities of the elderly due to immobility and frailty. Muscle degeneration is prominent in neuromuscular disorders [1] and acute muscle disuse conditions [2]. In these conditions discovery of the associated molecular mechanisms have led to better understanding of the pathophysiological processes of these disorders [3]. However, the molecular changes occurring in muscle degeneration in chronic conditions are still not fully understood. This is partly due to multifactorial etiologies, slow progression with aging, variation between individuals and unavailability of suitable models. Shoulder complaints are common in the general population [4-6], and their prevalence progresses during aging to more than $40 \%[7,8]$. Disease in the shoulder's rotator cuff (RC) muscles is characterized by disability of the shoulder joint, leading to pain and functional impairment in daily activities. The four RC muscles function as intricate motor units that dynamically stabilize the shoulder joint and provide arm mobility $[9,10]$. Initially, studies focused on the tendon degeneration [11-15], but recently muscle degeneration has been suggested to be primarily involved in affected RC muscles [16, 17]. Importantly, muscle degeneration in the shoulder is considered as a clinical determinant for surgical and non-surgical treatment outcomes $[16,18-20]$. However, variable and non- 
sustained outcomes call for better understanding of the intricate balance of shoulder pathology and functionality $[21,22]$.

Within the spectrum of chronic muscle disease tearing of the shoulder's RC is common. In RC tears the supraspinatus muscle (SSp) is initially affected and as the disease progresses, the subscapularis muscle (SSc) can tear as well [23]. In chronic RC diseases the deltoid muscle (DM) is clinically not affected, and can be considered a reference muscle [17]. Previously, we showed that while atrophy in the SSp and SSc continuously increase throughout adulthood, atrophy in the other RC muscles and the DM starts only from midlife onwards [17]. Furthermore, we found that torn RC muscles are characterized by an increase in extracellular matrix, and fatty droplets [17]. Despite high Pax-7 positive cells in torn muscles, their regeneration is limited [17]. Yet, the molecular mechanisms associated with these pathological conditions remain unknown.

Shoulder surgery provides a unique opportunity for ex-vivo investigations of functionally impaired muscles compared with unaffected muscles. Here we report a multidisciplinary study integrating radiological, histological, cellular and molecular investigations of diseased SSc and unaffected DM. We show that the SSc is highly degenerated and fibrotic but in contrast, viable muscle cell cultures were generated from both SSc and
DM. Muscle cell cultures of SSc were predominantly indifferent from DM. However, reduced myogenesis in SSc cultures was associated with down-regulation of genes regulating muscle development as well as up-regulation of extracellular region genes.

\section{RESULTS}

\section{Clinical characteristics}

Clinical features of diseased $\mathrm{RC}$ muscles were assessed from radiological imaging from 28 patients who underwent shoulder surgery. We compared RC muscle atrophy and fatty infiltration from patients with a diseased SSc with control subjects having an intact RC (Figure 1).

Patients with diseased SSc showed smaller crosssectional area (CSA) of the SSc and SSp (28\% and 32\% lower, ( $p<0.001$ in both) respectively), whereas the RC muscles infraspinatus (ISp) and teres minor (Tmi), and the adjacent DM were not significantly different in size compared with subjects with an intact RC. In the patient group fatty infiltration was present in all four RC muscles, with highest fatty infiltration in the SSc. Among all four $\mathrm{RC}$ muscles, the largest contrast of atrophy and fatty infiltration between the patient group and control subjects
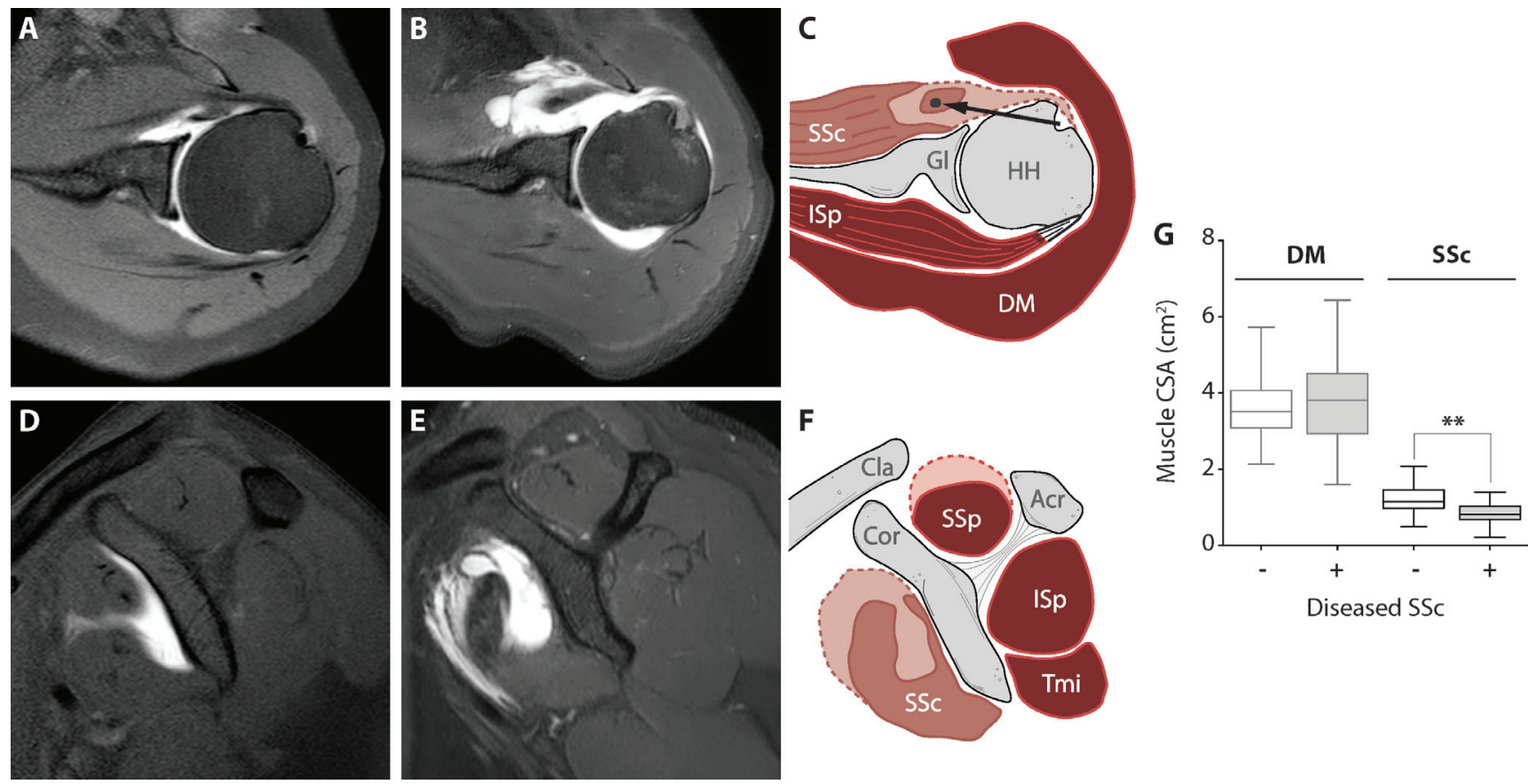

Figure 1: Clinical description of deltoid and subscapularis muscles. Anatomical landmarks of shoulder muscles in intact and torn conditions. Representative MRA images from the control group (panels A. and D., transversal and coronal view, respectively) and from torn SSc muscle (panels B. and E., transversal and sagittal view, respectively). A schematic illustration of muscle cross-sectional surface area (CSA) is shown in panels $\mathbf{C}$. and F. (transversal and coronal view, respectively). Muscle atrophy is indicated by the dashed line of the SSc. The long head of the biceps tendon is dislocated out of the bicipital groove, medially (black arrow). Subscapularis muscle (SSc), glenoid (Gl), humeral head (HH), infraspinatus (ISp), deltoid (DM), clavicle (cla), coracoid (cor), acromion (Acr), supraspinatus (SSp) and the teres minor (Tmi). Box plot $(\mathrm{G})$ shows CSA of the DM and SSC between control subjects $(\mathrm{n}=52)$ and patients with a diseased SSc $(\mathrm{n}$ $=28$ ). Statistical significance between DM and SSc using unpaired t-test: $* * p<0.001$. 


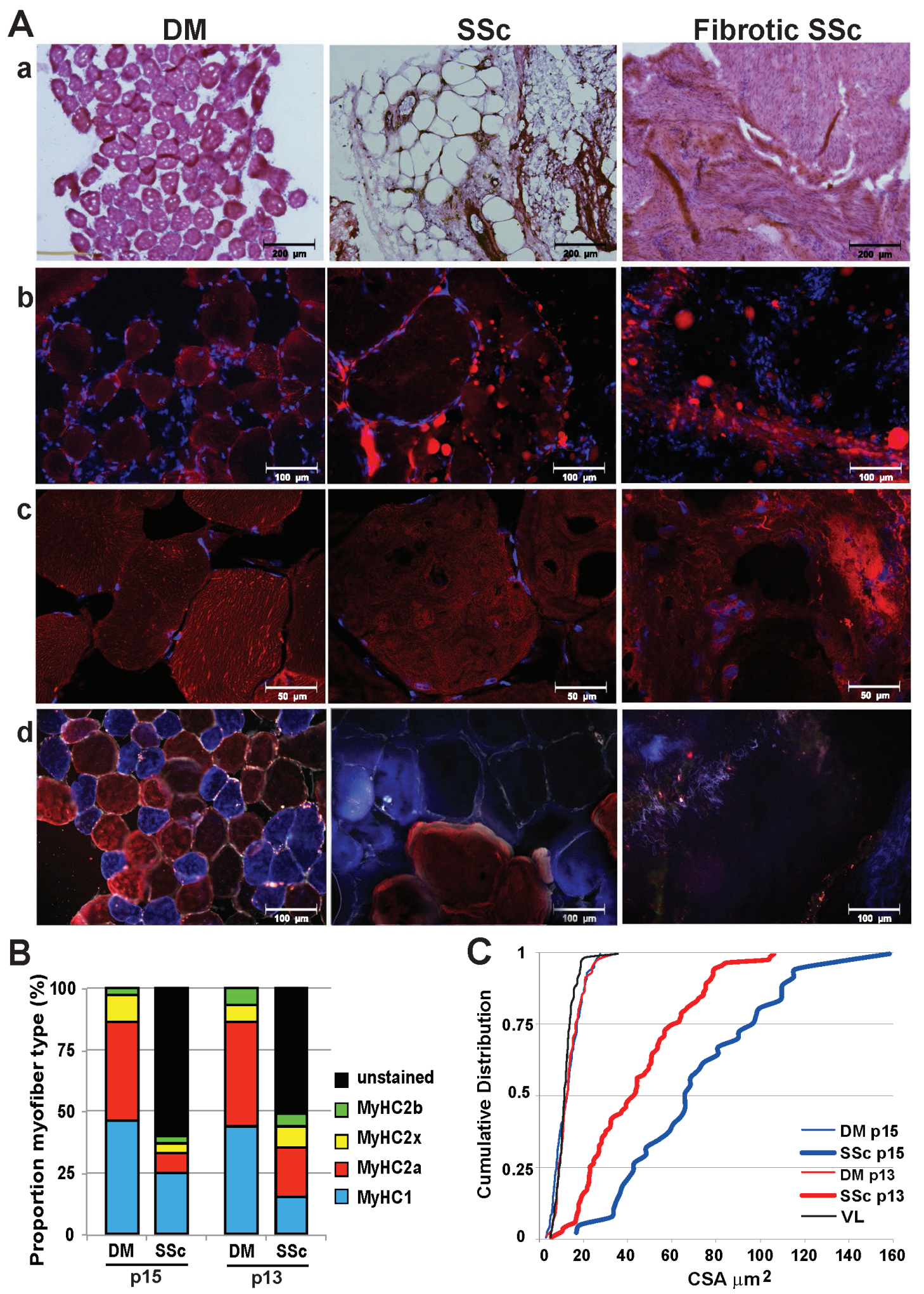

Figure 2: Histological analyses in deltoid and subscapularis muscles. A. Representative images of deltoid (DM) and subscapularis muscle (SSc) stained with: a. H\&E; b. Nile red (in red) for fatty droplets; c. C12-resazurin (in red) for oxidative metabolic activity. Nuclei are counterstained with DAPI in b and c ; d. Immunofluorescence with an antibody mix for MyHC-2b (green),-2a (red),-1 (blue) and laminin (white). For the SSc two conditions are shown: tissue containing myofibers (middle column) and highly fibrotic tissue without myofibers (right column). Images were taken with light microscope (a), fluorescence microscopes (b and d), or with a confocal microscope (c). Scale bars are 200 (a), 100 (b and d) or 50 (c) $\mu \mathrm{m}$. B. and C. Analyses in paired samples for DM and SSc in two patients. $\mathrm{B}$ : Bar charts depict proportion of myofibers expressing MyHC-isotypes and those that were unstained. C: Cumulative distribution plot of the CSA in SSc, DM and vastus lateralis (VL) reference. 
was found in the SSc (Table 1). In the DM, no fatty infiltration was found and CSA was similar in both groups (Table 1). Therefore for ex-vivo analyses we compared SSc with DM.

\section{Muscle histopathology of the SSc and DM}

Muscle tissue was first investigated with H\&E staining revealing that three out of five SSc samples were highly fibrotic and nearly no myofibers were present (Figure 2 and Table 2). In contrast, myofibers were found in all DM sections (Figure 2 and Table 2). Antilaminin immunohistochemistry confirmed the presence of myofibers in all DM samples, however only 2 out of $5 \mathrm{SSc}$ samples contained myofibers (Figure 2 and Table 2). Nile red staining showed the presence of large fatty droplets in the SSc, which were less common in the DM (Figure 2 and Table 2). This staining confirmed fatty infiltration found in radiological imaging.

Defects in metabolic activity, including glycogen catabolism, fatty acid oxidation, or mitochondrial oxidative phosphorylation often affect muscle function due to high energy consumptions [24]. In muscle wasting conditions oxidative metabolic activity can be changed [24]. Oxidative metabolic activity and oxidative phosphorylation can be monitored by an irreversible conversion of C12-resazurin to fluorescent resorufin, the fluorescent resorufin can record metabolic activity in the cell [25]. In undamaged deltoid muscles fluorescent foci of resorufin were scattered across the fibers, indicating active myofibers (Figure 2). However, in SSc muscles the distribution of fluorescent foci across myofibers was profoundly reduced (Figure 2). This indicates that oxidative metabolic activity and oxidative phosphorylation in the SSc are reduced.

We further investigated muscle pathology using the expression of myosin heavy chain (MyHC) isotypes. We applied an immunohistochemistry procedure with a mix of antibodies to MyHC-2b, $-2 \mathrm{a}$ and -1 isotypes to record fiber type distribution $[26,27]$. In both muscle tissues we found MyHC type-2a and type-1, but less frequent expression of MyHC-2b (Figure 2 and Table 2). In this immunostaining procedure non-stained fibers are regarded as MyHC-2x. To assess unstained myofibers, we then added an antibody to MyHC-2x. In DM samples all myofibers expressed one of the four MyHC isotypes (Figure S3), of which over $80 \%$ of the myofibers expressed either MyHC-2a or MyHC1 (Table 2). In the SSc muscles, however, over 50\% did not stain for any MyHC isotype. Of the myofibers that expressed MyHC, the majority expressed MyHC-1 (Table 2). Histological differences between diseased SSc and intact DM were further revealed in a paired analysis in the two patients with myofibers in the SSc. Above 50\% of myofibers lost MyHC expression (Figure 2B) and myofiber size was dramatically increased compared with DM in the same patient (Figure 2C).
A
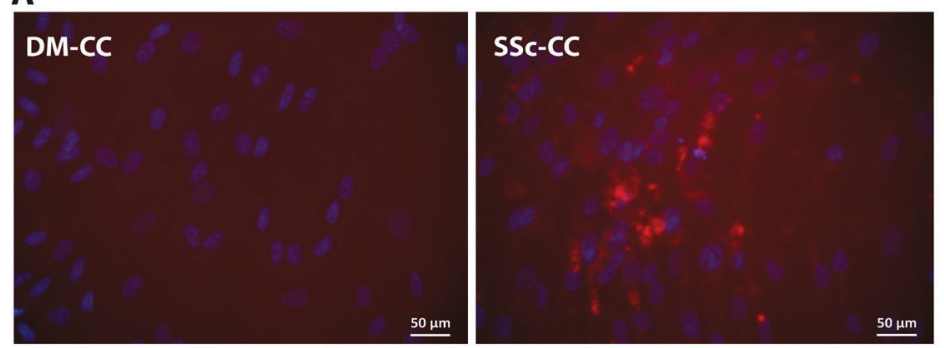

E
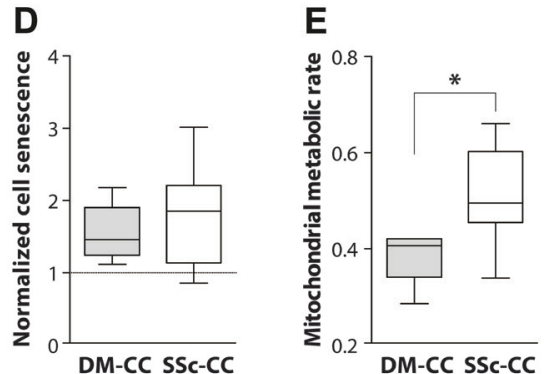

F

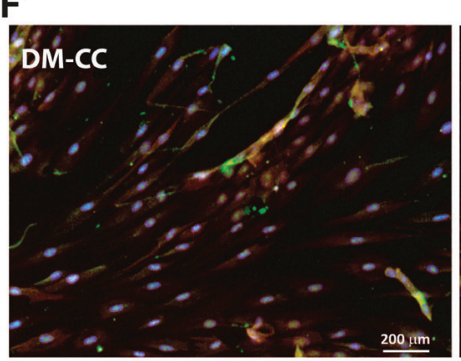

B

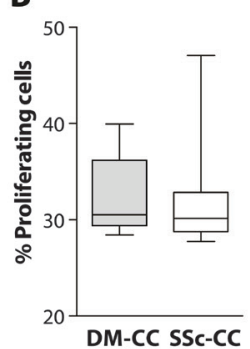

C

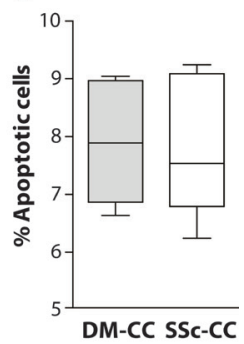

Figure 3: Cellular activities in deltoid (DM) and subscapularis (SSc) -derived muscle cell culture (CC). A. Nile red staining in deltoid (DM) and subscapularis muscle (SSc) cell culture (CC), nuclei are counterstained with DAPI (blue). Bar chart shows the percentage of cells containing fatty droplets. B. Bar chart shows the percentage of proliferating cells. C. Box plot shows the percentage of apoptotic cells. D. Box plot shows cell senescence (normalized to the reference vastus lateralis cell culture). E. Box plot shows the mitochondrial metabolic rate (normalized to the reference vastus lateralis cell culture). F. Representative images of fused cell cultures. Myoblasts are stained with Desmin (red) and fused cells are stained with MyHC (green). Nuclei are counterstained with DAPI (blue). Box plot shows the percentage of fused cells. Averages and standard deviations are from $\mathrm{n}=5$ (DM-CC) and $\mathrm{n}=8(\mathrm{SSc}-\mathrm{CC})$. Statistical significance between DM-CC and SSc-CC using unpaired t-tests: $* p<0.05, * * * p<0.001$. 
Table 1: Clinical characteristics

\begin{tabular}{|l|l|l|l|}
\hline Condition & $\begin{array}{l}\text { Control subjects } \\
(\boldsymbol{n}=\mathbf{5 2})\end{array}$ & $\begin{array}{l}\text { Patients with diseased } \\
\text { SSc }(\boldsymbol{n}=\mathbf{2 8})\end{array}$ & $\boldsymbol{p}$-value \\
\hline Age, years & $46.0(11.2)$ & $58.7(9.5)$ & $<\mathbf{0 . 0 0 1}$ \\
\hline Female, N (\%) & $27(52.9)$ & $11(39.3)$ & 0.25 \\
\hline & & & \\
\hline DM & & & \\
\hline CSA, $\mathrm{cm}^{2}$ & $35.9(7.9)$ & $38.3(11.6)$ & 0.27 \\
\hline Fatty infiltration, N (\%) & $0(0)$ & $0(0)$ & NA \\
\hline SSc & & & \\
\hline CSA, cm ${ }^{2}$ & $11.9(3.1)$ & $8.6(2.8)$ & $<\mathbf{0 . 0 0 1}$ \\
\hline Fatty infiltration, N (\%) & $0(0)$ & $22(78.6)$ & $<\mathbf{0 . 0 0 1}$ \\
\hline SSp & & & \\
\hline CSA, $\mathrm{cm}^{2}$ & $5.0(1.7)$ & $3.4(1.5)$ & $<\mathbf{0 . 0 0 1}$ \\
\hline Fatty infiltration, N (\%) & $8(15.7)$ & $20(71.4)$ & $<\mathbf{0 . 0 0 1}$ \\
\hline ISp & & & 0.61 \\
\hline CSA, $\mathrm{cm}^{2}$ & $7.2(1.8)$ & $7.0(2.3)$ & $<\mathbf{0 . 0 0 1}$ \\
\hline Fatty infiltration, N (\%) & $8(15.7)$ & $19(67.9)$ & \\
\hline Tmi & & & 0.68 \\
\hline CSA, cm ${ }^{2}$ & $3.7(1.2)$ & $3.6(1.2)$ & $\mathbf{0 . 0 0 1}$ \\
\hline Fatty infiltration, N (\%) & $0(0)$ & $6(21.4)$ & \\
\hline
\end{tabular}

Means (standard deviations) are provided unless otherwise stated. Deltoid (DM), cross sectional area (CSA), subscapularis (SSc), supraspinatus (SSp), infraspinatus (ISp), teres minor (Tmi). The $\mathrm{p}$-values are calculated with unpaired t-tests, or chi-squared tests where appropriate.

Table 2: Histological observations in deltoid and subscapularis muscles

\begin{tabular}{|c|c|c|}
\hline & Deltoid $(n=7)$ & Subscapularis $(n=5)$ \\
\hline Myofibers in muscle & $7 / 7$ & $2 / 5$ \\
\hline Fatty droplets & $2 / 7$ & $5 / 5$ \\
\hline Fiber CSA, $\wedge^{\wedge} \mu \mathrm{m}^{2}$ & $62.1(17.5)$ & $105.8(22.6)$ \\
\hline MyHC-2b, ^\% $\%$ & $8.1(6.9)$ & $5.0(3.7)$ \\
\hline MyHC- $2 \mathrm{x}, \wedge \%$ & $9.6(7.2)$ & $7.0(3.0)$ \\
\hline MyHC-2a, ^\% & $42.9(13.8)$ & $12.5(10.6)$ \\
\hline МyHC-1, ^\% & $40.1(12.2)$ & $20.0(7.1)$ \\
\hline Unstained fibers, $\wedge \%$ & $0(0)$ & $55.0(6.0)$ \\
\hline
\end{tabular}

Analysis per sample shows the number of positive samples out of total muscle samples. Analyses carried out only from myofiber-containing sections in the subscapularis are depicted with $\wedge$. Fully degenerated muscles, as shown in Figure 2, were excluded from these analyses. Means (standard deviations) are provided unless otherwise stated. Cross sectional area (CSA), myosin heavy chain (MyHC). 


\section{Cellular analyses of SSc- and DM-derived myoblasts}

To uncover the intrinsic molecular and cellular mechanisms underlying pathophysiological changes in chronic RC muscles, we generated muscle cell cultures from SSc muscles and Del as control. We applied this approach as the histology of chronically degenerated SSc muscle indicated that the tissue is highly fibrotic. Fatty droplets were prevalent in the SSc muscle cell cultures but were absent in the DM muscle cell cultures (Figure 3A). Within the desmin-positive cell population, myoblasts from the SSc and DM muscle cell cultures did not differ with respect to cell proliferation, apoptosis or cell senescence (Figure 3B-3E). The mitochondrial metabolic rate of the $\mathrm{SSc}$ myoblast cell cultures was higher than the DM myoblast cultures (Figure 3F). Moreover, we found that cell fusion in SSc derived cell culture was significantly lower compared with DM cultures (Figure 3G).

B

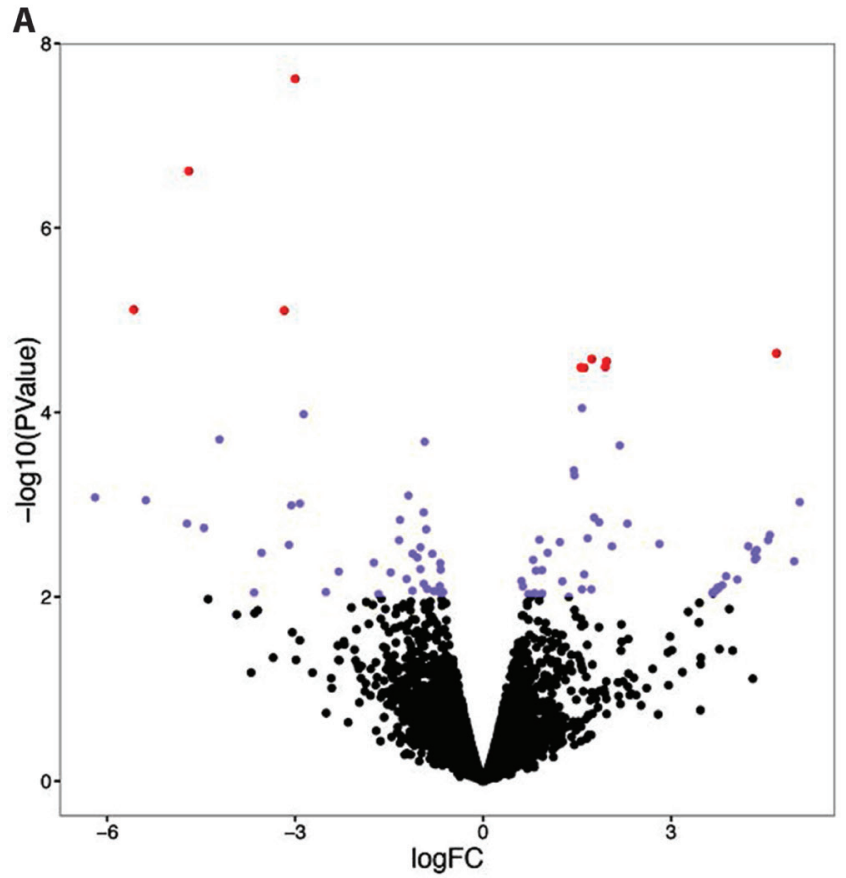

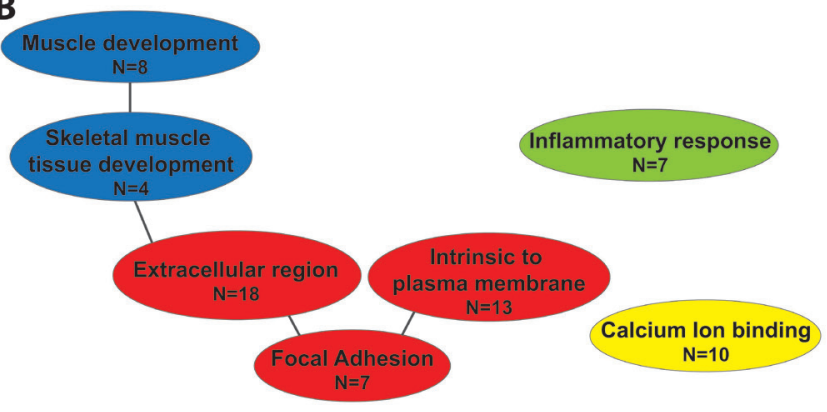

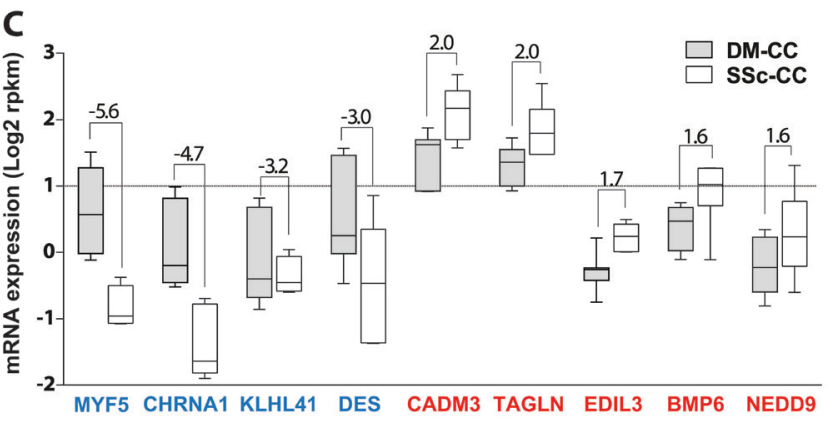

D

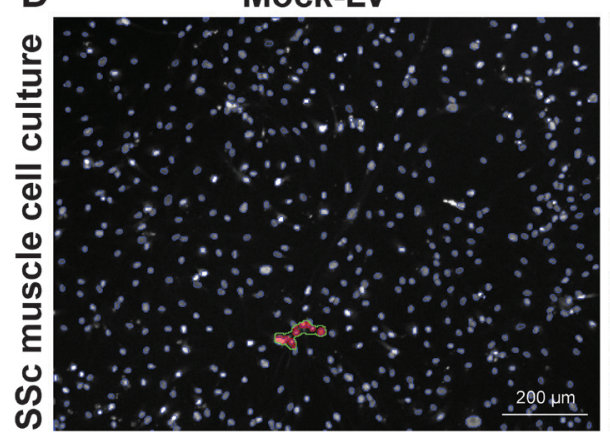

MyoD-LV

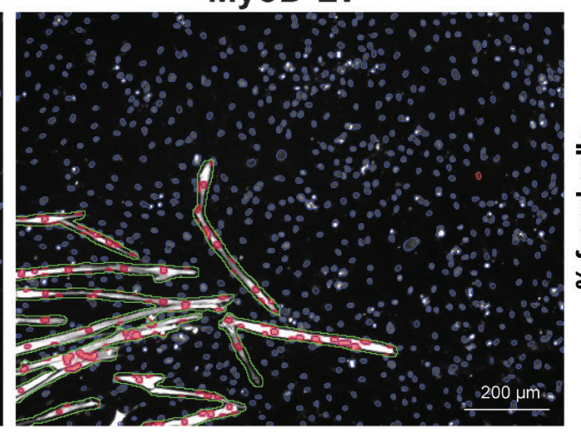

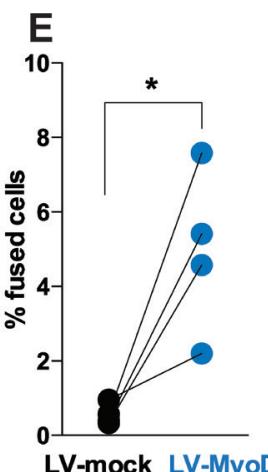

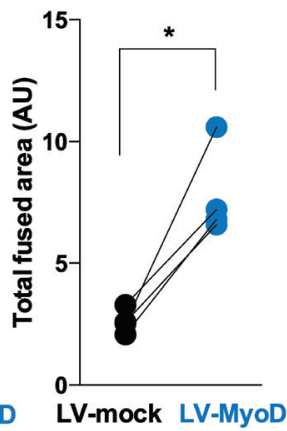

Figure 4: RNA expression profiles in deltoid and subscapularis muscle-derived cell cultures. A. Volcano plot shows log2 fold change (FC) versus - $\log 10$ of the $\mathrm{p}$-value of all genes found in the RNA-seq (pair-wise analyses, $\mathrm{n}=7$ ). Red dots indicate significantly dysregulated genes $(p<0.05$ FDR). Blue dots indicate dysregulated genes with a nominal $p$-value $<0.01$. Positive or negative FC indicates higher or lower expression in subscapularis ( $\mathrm{SSc}$ ) compared with the deltoid (DM) cell cultures (CC). B. Functional gene ontology (GO) of dysregulated genes $(p<0.01)$, daughter GO clusters are connected with a line. GO clusters of muscle development are depicted in blue, in red the extracellular region, in green the inflammatory system and in yellow calcium binding. $\mathrm{N}$ indicates the number of genes. C. Box plot shows expression levels in SSc-CC or DM-CC for the most significantly dysregulated genes $(p<0.05$ FDR). Fold changes between DM and SSc are depicted. Genes of the GO muscle development are marked in blue and those of the extracellular region in red. D. Representative images of fused SSc cell cultures transduced with either mock or MyoD lentivirus (LV). Myoblasts are stained with MyHC (white) and segmented in green. Nuclei are counterstained with DAPI (blue) and segmented in blue. Nuclei within segmented MyHC objects are marked in red. Scale bar is $200 \mu \mathrm{m}$. E. Plots show paired analyses of \% of fused cells and total fused area between mock and MyoD LV in 4 SSc cell cultures. *: $p$-value $<0.05$, using a paired T-test. 


\section{RNA expression profiles in myoblasts}

Next we assessed molecular differences between the SSc and DM derived muscle cell cultures using RNA-seq in seven patients. A paired analysis of RNA-seq data from the two muscles of the same patient revealed only few molecular differences between the SSc and DM myoblasts (Figure 4A). Ultimately, using a false discovery rate (FDR) of 0.05 , only 14 genes were identified as differentially expressed between the SSAC- and DM- derived cultures (114 with nominal $p$-value $<0.01$ ) (Figure 4A). The 114 dysregulated genes were clustered into four functional gene-ontology groups: the extracellular region $(N=18)$ and focal adhesion $(N=7)$, calcium ion binding $(N=10)$, inflammatory response $(N=7)$ and four genes clustered in skeletal muscle development (Figure 4B). The most significantly dysregulated genes ( $p<0.05$ FDR) affecting muscle cell development were down-regulated, while genes within the extracellular region, were up-regulated in the SSc myoblast cultures (Figure 4C). The expression level of muscle stem cells genes did not differ between these two myoblast cultures (Figure S4).

To confirm that reduced expression of myogenic genes causes reduced cell fusion in SSc myoblasts cultures, we elevated MyoD expression levels using lentivirus transduction into SSc cultures, and mock virus was used as control. We assessed the myogenesis using expression of MyHC. We found that overexpression of MyoD restored myogenesis in the SSc cultures (Figure $4 \mathrm{D})$. In average myogenesis was restored with an increase of $4.2 \%$ of fused cells $(p<0.05$, paired T-test) and a 3.1 fold larger fused area ( $p<0.05$, paired T-test) (Figure 4E).

\section{DISCUSSION}

Chronic muscle degeneration is highly prevalent in the elderly, characterized by loss of muscle mass and muscle strength, and leads to immobility and abrogates functionality and independence. In contrast to muscle waste in acute conditions, such as muscle disuse in denervation conditions [28] or starvation stress [29], the molecular mechanisms that are affected in chronic conditions are poorly understood. We present a multidisciplinary study revealing the most affected molecular mechanisms in degenerated muscles of chronic conditions, focusing on shoulder muscles. We chose to study shoulder muscles as the mechanical stresses on muscles around this joint are comparable. Furthermore, during shoulder surgery we could obtain two muscles, diseased and healthy, from each patient and those were paired analyzed.

We show that diseased SSc muscles are loaded with fatty infiltration, fibrosis, low metabolic activity, and reduced expression of MyHC isotypes. This is in agreement with our recent study showing that torn ISp muscle show similar histological marks including fatty infiltration, fibrosis and reduced expression of MyHC isotypes [17]. Reduced oxidative metabolism can suggest impaired mitochondrial activity [25], which is often found in degenerated muscles [24]. Moreover, in torn ISp we found a profound increase in collagen I staining, marking thickening of the extracellular matrix. This observation agrees with up-regulation of the extracellular matrix functional group we report here in torn SSc. Extracellular thickening is among the histological marks of chronic muscle degeneration and fibrosis [17, 30, 31]. In addition, we found that genes of the muscle functional group, specifically myogenesis genes, are down-regulated in cell culture from SSc compared with DM cell culture. This observation is in agreement with reduced myogenesis in the SSc cell culture. Moreover, overexpression of MyoD in SSC culture, which was found to be down-regulated, caused an increase in cell fusion. Restoring levels of additional genes from the muscle development group, and supressing expression of ECM genes could potentially lead to enhance muscle regeneration in chronic conditions.

Despite the pronounced histological changes in diseased SSc muscle, generation of myoblast cell culture from the diseased SSc was as successful as from the DM. Myoblasts cultures from degenerated SSc differed from DM cultures by only limited cellular and molecular signatures. We found no differences in cell proliferation, senescence and apoptosis between cell cultures from diseased SSc and DM. This observation is in agreement with a recent study showing no differences in cell proliferation between SSp and DM muscles [32]. In the torn SSp cell fusion was unaffected [32]. We, however, found reduced myogenesis in the torn SSc. This difference can be explained by differences in RC pathology between these two studies. A tear limited to the SSp is considered less severe $\mathrm{RC}$ disease, compared to tears also involving the SSc $[8,9,33]$. We therefore suggest that in more severe RC disease, muscle pathology worsens and cells have reduced myogenic capacity.

In chronically diseased SSc or ISp muscles [17] reduced expression of MyHC isotypes was complemented with an increase in CSA of myofibers. Genetic loss of MyHC expression causes an increased myofiber CSA [34]. This suggests that in chronically degenerated muscles reduced expression of MyHC isotypes could cause loss of myofibers construction and hence muscle weakness. It remains to be investigated how torn muscle conditions lead to loss of MyHC expression. In contrast to the chronic conditions investigated here, in animal models for RC tear that were generated by acute conditions (e.g. denervation, chemically, mechanically) MyHC expression is unaffected [35-40]. This suggests that molecular mechanisms associated with acute tear conditions differ from those in chronically diseased muscles.

Our results suggest that in chronically diseased $\mathrm{SSc}$, muscle regeneration could be impaired due to 
reduced myogenesis of the muscle stem cells in an environment of thickened extracellular matrix. Targeting the extracellular region and muscle development could open new options for therapeutic developments such as cell therapies to enhance muscle regeneration. Repression of the extracellular region could be combined with surgical repair. Transplantation of in vitro propagated myoblasts into the surgical site could support muscle regeneration towards cell based therapy development. Cell therapy could ultimately enhance muscle regeneration in a combinatorial treatment to prevent muscle re-tear. Towards this, a recent study demonstrated grafting and fusion of satellite cells from non-degenerated muscles into injured muscles in mice [32]. In addition, efforts to reduce the extracellular region are developed in models for Duchenne muscular dystrophy [31], and those could be also applied for shoulder patients to order to regenerate myofibers.

This is an explorative study, with a small patient group and data have to be interpreted with caution. However, paired analyses increase statistical power and thus led to significant results. An independent replication study to validate our results should be performed. In this study we used muscle samples from shoulder pathology at advanced stage. In this stage the SSp is mostly torn, therefore the results presented here are an underestimation of muscle degeneration affecting only the SSp, which is more prevalent but less severe compared to tears extending to the SSc $[8,9,33]$. The more severe SSc pathology gives a broad spectrum to evaluate pathophysiological changes in chronic and torn muscles.

\section{MATERIALS AND METHODS}

In this explorative study redundant muscle samples were collected as waste material from eleven patients that underwent shoulder surgery at the Leiden University Medical Center between 2012 and 2013 for ex-vivo analyses. All muscles samples were collected according to the Medical Ethical regulations of the institutional ethic review board of the Leiden University Medical Center and according to the guideline 'good use of redundant tissue for clinical research' by the Dutch Federation of Medical Research Societies. Anonymous use of redundant tissues as waste material for research purposes is according to a standard agreement with patients in our hospital.

\section{Surgical procedure and muscle collection}

For shoulder surgery, patients were positioned in a beach chair pose and prepared and draped in a sterile fashion for shoulder surgery under general anesthesia. All patients received standard antibiotic prophylaxis before surgery. Due to damage of the SSc muscle, waste material for sampling could easily be obtained. In all cases the diseased SSc muscle was detached from the insertion site on the greater tubercle. Additionally, DM muscle samples (about $2 \mathrm{~mm}^{3}$ ) were collected, which resulted from splitting the DM and pectoral muscle for the surgical approach. One muscle sample of each muscle was immediately frozen in liquid nitrogen and was stored at $-80^{\circ} \mathrm{C}$ prior to the histological analyses, and the second sample was dipped into $100 \%$ Fetal Calf Serum (FCS) + $50 \mu \mathrm{g} / \mathrm{ml}$ gentamycin for the establishment of myoblast cell cultures. The myoblast cell cultures were generated as described in [41] after 7-10 day in vitro propagation cultures reached $80-95 \%$ confluence in a 6 well plate and cells were kept frozen in liquid nitrogen.

\section{Histology and imaging}

From seven DM and five SSc frozen samples were cryosectioned $(20 \mu \mathrm{m})$ on dry ice using the Leica CM3050-S cryosection (Leica, Solms, Germany). Other samples were too small for cryosectioning. Histological analyses were: 1- hematoxylin and eosin (H\&E) staining (Standard Operating Procedure: MDC1A_M.1.2.004). 2- myofiber typing protocol is detailed in Supplementary Methods file. 3- Fatty droplets staining with $1 \mu \mathrm{M}$ Nile red (Sigma-Aldrich, Saint Louis, Missouri, USA) followed by PBS washing. Nuclei were counterstained with DAPI. 4- Oxidative metabolic activity was assessed with $10 \mathrm{mM}$ C12-Resazurin (Invitrogen) incubated for 15 minutes and followed by extensive washes with 50\% methanol in PBS.

All slides were stained at the same day with the same mix. Slides were mounted with Polymount (Polyscience). C12-Resazurin fluorescence was visualized with a TCS-SP5 confocal microscope. Visualization of H\&E stained sections was carried out with a Leica DFC550 light microscope. Fluorescent signals, otherwise, were captured with DM5500 microscope (Leica) using LAS AF software V2.3.6. or with Array scan VTI HCA (Thermofisher, Waltham, Massachusetts, USA). For each muscle, an unstained section was used to determine autofluorescence. Vastus lateralis (VL) cross-sections were used as a reference for each procedure and standardization of imaging. Per fluorophore, all muscle sections were imaged with the same configuration and exposure at the same day. Image overlay and image processing was carried out with Leica LAS AF version 2.6.0 build 7266. Image quantification was carried out with ImageJ (http:// rsbweb.nih.gov).

\section{Analyses of myoblast cell cultures}

For cellular and molecular analyses myoblast cell cultures were seeded simultaneously. Cellular analyses were carried out on cultures at passage 1 from eight SSc samples and five DM samples that reached 20,000 cells at the same time. Muscle cell fusion and cell senescence 
were carried out on cells at passage 2. Cellular analyses are schematically summarized in Supplementary Figure S1. RNA-seq was carried out on seven paired cultures at passage 2 . The researchers were blinded to the muscle type during experimentation and analyses. The cellular analyses were performed as summarized and detailed in the Supplementary Methods file.

\section{RNA isolation and RNA analyses}

Cultures from seven pairs were included in the final analysis after passing control quality (Supplementary Table S1). RNA was isolated from cultures at passage 2 grown in a 12 well plate using the Nucleospin RNA II (Macherey-Nagel, Düren, Germany) according to the instructions of the manufacturer, and was stored at $-80^{\circ} \mathrm{C}$ prior to analyses. RNA quality was assessed using the Agilent 2100 Bioanalyzer (Agilent Technologies, Santa Clara, California, USA). RNAseq library preparation and pair-wise analysis to identify SSc dysregulated genes is detailed in the Supplementary Methods file.

\section{Lentiviral transduction}

4 cultures of SSc at passage 2 were transduced with MyoD lentivirus [42] or control lentivirus (multiplicity of infection (MOI): 5 . After 3 days cells were incubated at fusion conditions, 2\% horse-serum in DMEM, for 5 days. Quantification of fused cells was carried out as detailed in the Supplementary Methods file. An average of 9158 nuclei per condition was included.

\section{Radiological imaging}

Due to medical ethical constrains we were not allowed to associate muscle samples with medical information, therefore all MRIs of patients undergoing shoulder surgery involving SSc muscle damage $(n=$ 28) between 2012 and 2013 were evaluated. Patients received local intra-articular analgesia and a contrast agent for arthrography via a posterior approach. Magnetic Resonance imaging with arthrography (MRA) scanning and analyses were performed as previously detailed [9, 17]. As control group, 52 subjects from a previous study were included with structurally intact RC muscles [9]. The MRI scan of one control could not be reviewed. The institutional ethics review board waived the need for participant informed consent to review the MRIs. The MRIs were anonymized and observers were blinded for outcomes.

\section{Statistical analyses}

All statistical analyses were performed using IBM SPSS statistics version 20 (IBM Corp, 2011, Armonk, New York, USA). Categorical data are expressed using absolute values and percentages. Parametric continuous data are described with means and 95\%-confidence intervals $(95 \%$-CI). Statistical assessments were carried out with unpaired Student's t-tests, unless otherwise stated.

\section{FUNDING}

This work is supported by the Dutch Arthritis Association (DAA), grant number RF 13-1-303.

\section{ACKNOWLEDGMENTS}

The authors thank dr. Stephen Tapscott for kindly providing the MyoD lentivirus. Author contributions: Biopsies were collected by JFH, AK and JN, NEV maintained the cell cultures, cellular analyses were performed by YR and VR, histological staining were performed by NKG, RNA isolation was performed by AK and VR, RNA-seq analyses were performed by ZT, WA, SMK and PACH, this study was set up by RGHH and VR, YR and JFH drafted the manuscript and all authors contributed to the discussion of the results.

\section{CONFLICTS OF INTERESTS}

The authors declare no conflicts of interest.

\section{REFERENCES}

1. Krause S. Insights into muscle degeneration from heritable inclusion body myopathies. Front Aging Neurosci. 2015; $7: 13$.

2. Wall BT, Dirks ML and van Loon LJ. Skeletal muscle atrophy during short-term disuse: implications for agerelated sarcopenia. Ageing ResRev. 2013; 12:898-906.

3. Sandri M, Coletto L, Grumati $\mathrm{P}$ and Bonaldo $\mathrm{P}$. Misregulation of autophagy and protein degradation systems in myopathies and muscular dystrophies. JCell Sci. 2013; 126:5325-5333.

4. Greving K, Dorrestijn O, Winters JC, Groenhof F, van der Meer K, Stevens M and Diercks RL. Incidence, prevalence, and consultation rates of shoulder complaints in general practice. ScandJRheumatol. 2012; 41:150-155.

5. Picavet HS and Schouten JS. Musculoskeletal pain in the Netherlands: prevalences, consequences and risk groups, the DMC(3)-study. Pain. 2003; 102:167-178.

6. Roquelaure Y, Ha C, Leclerc A, Touranchet A, Sauteron M, Melchior M, Imbernon E and Goldberg M. Epidemiologic surveillance of upper-extremity musculoskeletal disorders 
in the working population. Arthritis Rheum. 2006; 55:765778.

7. Sher JS, Uribe JW, Posada A, Murphy BJ and Zlatkin MB. Abnormal findings on magnetic resonance images of asymptomatic shoulders. JBone Joint SurgAm. 1995; 77:1015.

8. Mall NA, Kim HM, Keener JD, Steger-May K, Teefey SA, Middleton WD, Stobbs G and Yamaguchi K. Symptomatic progression of asymptomatic rotator cuff tears: a prospective study of clinical and sonographic variables. JBone Joint SurgAm. 2010; 92:2623-2633.

9. Henseler JF, Raz Y, Nagels J, van Zwet EW, Raz V and Nelissen RG. Multivariate analyses of rotator cuff pathologies in shoulder disability. PLoSOne. 2015; 10:e 0118158.

10. Henseler JF, de Witte PB, de Groot JH, van Zwet EW, Nelissen RG and Nagels J. Cranial translation of the humeral head on radiographs in rotator cuff tear patients: the modified active abduction view. MedBiolEng Comput. 2014; 52:233-240.

11. Klatte-Schulz F, Pauly S, Scheibel M, Greiner S, Gerhardt C, Schmidmaier G and Wildemann B. Influence of age on the cell biological characteristics and the stimulation potential of male human tenocyte-like cells. EurCell Mater. 2012; 24:74-89.

12. Boileau P, Brassart N, Watkinson DJ, Carles M, Hatzidakis AM and Krishnan SG. Arthroscopic repair of full-thickness tears of the supraspinatus: does the tendon really heal? JBone Joint SurgAm. 2005; 87:1229-1240.

13. Pauly S, Stahnke K, Klatte-Schulz F, Wildemann B, Scheibel $M$ and Greiner S. Do patient age and sex influence tendon cell biology and clinical/radiographic outcomes after rotator cuff repair? AmJSports Med. 2015; 43:549-556.

14. Castagna A, Cesari E, Gigante A, Conti M and Garofalo R. Metalloproteases and their inhibitors are altered in both torn and intact rotator cuff tendons. MusculoskeletSurg. 2013; 97 Suppl 1:39-47.

15. Sejersen MH, Frost P, Hansen TB, Deutch SR and Svendsen SW. Proteomics perspectives in rotator cuff research: a systematic review of gene expression and protein composition in human tendinopathy. PLoSOne. 2015; 10:e0119974.

16. Laron D, Samagh SP, Liu X, Kim HT and Feeley BT. Muscle degeneration in rotator cuff tears. JShoulderElbowSurg. 2012; 21:164-174.

17. Raz Y, Henseler JF, Kolk A, Riaz M, van der Zwaal P, Nagels J, Nelissen RGHH and Raz V. Patterns of ageassociated degeneration differ in shoulder muscles. FrontAging Neurosci. 2015; 7:236.

18. Gladstone JN, Bishop JY, Lo IK and Flatow EL. Fatty infiltration and atrophy of the rotator cuff do not improve after rotator cuff repair and correlate with poor functional outcome. AmJSports Med. 2007; 35:719-728.

19. Kim JR, Cho YS, Ryu KJ and Kim JH. Clinical and radiographic outcomes after arthroscopic repair of massive rotator cuff tears using a suture bridge technique: assessment of repair integrity on magnetic resonance imaging. AmJSports Med. 2012; 40:786-793.

20. Shen PH, Lien SB, Shen HC, Lee CH, Wu SS and Lin LC. Long-term functional outcomes after repair of rotator cuff tears correlated with atrophy of the supraspinatus muscles on magnetic resonance images. JShoulderElbowSurg. 2008; 17:1S-7S.

21. Oh LS, Wolf BR, Hall MP, Levy BA and Marx RG. Indications for rotator cuff repair: a systematic review. ClinOrthopRelat Res. 2007; 455:52-63.

22. Lambers Heerspink FO, van Raay JJ, Koorevaar RC, van Eerden PJ, Westerbeek RE, van 't RE, van dA-S, I and Diercks RL. Comparing surgical repair with conservative treatment for degenerative rotator cuff tears: a randomized controlled trial. J ShoulderElbowSurg. 2015; 24:1274-1281.

23. Denard PJ and Burkhart SS. Arthroscopic recognition and repair of the torn subscapularis tendon. ArthroscTech. 2013; 2:e373-e379.

24. Karakelides H and Nair KS. Sarcopenia of aging and its metabolic impact. CurrTopDevBiol. 2005; 68:123-148.

25. Talbot JD, Barrett JN, Barrett EF and David G. Rapid, stimulation-induced reduction of C12-resorufin in motor nerve terminals: linkage to mitochondrial metabolism. JNeurochem. 2008; 105:807-819.

26. Gregorevic P, Meznarich NA, Blankinship MJ, Crawford RW and Chamberlain JS. Fluorophore-labeled myosinspecific antibodies simplify muscle-fiber phenotyping. Muscle Nerve. 2008; 37:104-106.

27. Schiaffino $\mathrm{S}$ and Reggiani C. Fiber types in mammalian skeletal muscles. Physiol Rev. 2011; 91:1447-1531.

28. Verdijk LB, Dirks ML, Snijders T, Prompers JJ, Beelen M, Jonkers RA, Thijssen DH, Hopman MT and Van Loon LJ. Reduced satellite cell numbers with spinal cord injury and aging in humans. Medicine and science in sports and exercise. 2012; 44:2322-2330.

29. Schiaffino S, Dyar KA, Ciciliot S, Blaauw B and Sandri M. Mechanisms regulating skeletal muscle growth and atrophy. The FEBS journal. 2013; 280:4294-4314.

30. Raz V, Sterrenburg E, Routledge S, Venema A, van der Sluijs BM, Trollet C, Dickson G, van Engelen BG, van der Maarel SM and Antoniou MN. Nuclear entrapment and extracellular depletion of PCOLCE is associated with muscle degeneration in oculopharyngeal muscular dystrophy. BMCNeurol. 2013; 13:70.

31. Mann CJ, Perdiguero E, Kharraz Y, Aguilar S, Pessina P, Serrano AL and Munoz-Canoves P. Aberrant repair and fibrosis development in skeletal muscle. SkeletMuscle. $2011 ; 1: 21$.

32. Meyer GA, Farris AL, Sato E, Gibbons M, Lane JG, Ward SR and Engler AJ. Muscle progenitor cell regenerative capacity in the torn rotator cuff. JOrthopRes. 2015; 33:421429. 
33. Gerhardt C, Lehmann L, Lichtenberg S, Magosch P and Habermeyer P. Modified L'Episcopo tendon transfers for irreparable rotator cuff tears: 5-year follow-up. ClinOrthopRelat Res. 2010; 468:1572-1577.

34. Allen DL, Harrison BC, Sartorius C, Byrnes WC and Leinwand LA. Mutation of the IIB myosin heavy chain gene results in muscle fiber loss and compensatory hypertrophy. AmJPhysiol Cell Physiol. 2001; 280:C637-C645.

35. Rowshan K, Hadley S, Pham K, Caiozzo V, Lee TQ and Gupta R. Development of fatty atrophy after neurologic and rotator cuff injuries in an animal model of rotator cuff pathology. JBone Joint SurgAm. 2010; 92:2270-2278.

36. Bonaldo $\mathrm{P}$ and Sandri M. Cellular and molecular mechanisms of muscle atrophy. DisModelMech. 2013; 6:25-39.

37. Ruegg MA and Glass DJ. Molecular mechanisms and treatment options for muscle wasting diseases. AnnuRevPharmacolToxicol. 2011; 51:373-395.

38. Itoigawa $\mathrm{Y}$, Kishimoto KN, Sano H, Kaneko K and Itoi E. Molecular mechanism of fatty degeneration in rotator cuff muscle with tendon rupture. JOrthopRes. 2011; 29:861-866.

39. Frey E, Regenfelder F, Sussmann P, Zumstein M, Gerber C, Born W and Fuchs B. Adipogenic and myogenic gene expression in rotator cuff muscle of the sheep after tendon tear. JOrthopRes. 2009; 27:504-509.

40. Gumucio JP, Korn MA, Saripalli AL, Flood MD, Phan AC, Roche SM, Lynch EB, Claflin DR, Bedi A and Mendias CL. Aging-associated exacerbation in fatty degeneration and infiltration after rotator cuff tear. JShoulderElbowSurg. 2014; 23:99-108.

41. Bigot A, Klein AF, Gasnier E, Jacquemin V, Ravassard P, Butler-Browne G, Mouly V and Furling D. Large CTG repeats trigger p16-dependent premature senescence in myotonic dystrophy type 1 muscle precursor cells. AmJPathol. 2009; 174:1435-1442.

42. Yao Z, Fong AP, Cao Y, Ruzzo WL, Gentleman RC and Tapscott SJ. Comparison of endogenous and overexpressed MyoD shows enhanced binding of physiologically bound sites. SkeletMuscle. 2013; 3:8. 